\title{
Towards chaos criterion in quantum field theory.
}

\author{
V.I. Kuvshinov ${ }^{1}$, A.V. Kuzmin ${ }^{2}$ \\ 1-Institute of Physics, 220072 Belarus, Minsk, Scorina,68. \\ Tel. 375-172-84-16-28, fax: 375-172-84-08-79. \\ E-mail: kuvshino@dragon.bas-net.by \\ 2-Institute of Physics, 220072 Belarus, Minsk, Scorina,68. \\ E-mail: avkuzmin@dragon.bas-net.by
}

\begin{abstract}
Chaos criterion for quantum field theory is proposed. Its correspondence with classical chaos criterion in semi-classical regime is shown. It is demonstrated for real scalar field that proposed chaos criterion can be used to investigate stability of classical solutions of field equations.
\end{abstract}

Phenomenon of chaos attracts much attention in various fields of physics. Originally it was associated with problems of classical mechanics and statistical physics. Substantiation of statistical mechanics initiated intensive study of chaos and uncovered its basic properties mainly in classical mechanics. One of the main results in this direction was a creation of KAM theory and understanding of the phase space structure of Hamiltonian systems [1], [2]. It was clarified that the root of chaos was local instability of dynamical system [3]. Local instability leads to mixing of trajectories in phase space and thus to non-regular behavior of the system and chaos [4]-[G]. Significant property of chaos is its prevalence in various natural phenomena. It explains a large number of works in this field.

Large progress is achieved in understanding of chaos in semi-classical regime of quantum mechanics via analysis of the spectral properties of the system [6], [7]. Semi-classical restrictions are important, because a large number of energy levels in small energy interval is needed to provide a certain statistics $[8]$.

Investigation of the stability of classical field solutions faces difficulties caused by infinite number of degrees of freedom. That is why authors often restrict their consideration by investigation of some model field configurations [9].

There are papers devoted chaos in quantum field theory [10]. But there is no generally recognized definition of chaos for quantum fields [11]. This fact restricts use of chaos theory in field of elementary particle physics. At the same time it is well known that field equations of all four types of fundamental interactions have chaotic solutions [12] and high energy physics reveales the phenomenon of intermittency [13]. The aim of this work is to propose chaos criterion applied for both quantum mechanical and quantum field systems.

The paper is structured as follows. At first we generalize classical Toda criterion of local instability [14]-[16] for systems with any finite number of degrees of freedom. Then using analogy with statistical mechanics we formulate chaos criterion for quantum mechanics and 
quantum field theory. After that we apply formulated chaos criterion to quantum mechanical systems in semi-classical approximation and check its accordance with generalized Toda criterion for the corresponding classical system (correspondence principle). And finally we apply formulated chaos criterion to analyze $\varphi^{4}$-theory of real scalar field.

Toda criterion of local instability for classical mechanical systems was at first formulated in 14. It was reformulated for Hamiltonian systems with two degrees of freedom by Salasnich [15]. Local instability of classical conservative Hamiltonian system with finite number of freedoms and finite (at finite energy) available phase space volume leads to mixing, destruction of the first integrals of motion and all that one calls chaos [4]-6]. Agreement between Toda criterion and criterion of classical chaos based on KAM theory and conception of nonlinear resonance was checked on the particular example in 17.

Consider classical Hamiltonian system with any finite number of degrees of freedom

$$
H=\frac{1}{2} \vec{p}^{2}+V(\vec{q}) \quad, \quad \vec{p}=\left(p_{1, \ldots, p_{N}}\right) ; \vec{q}=\left(q_{1, \ldots,}, q_{N}\right), \quad N>1
$$

Behavior of the classical system is locally unstable if distance between two neighboring trajectories grows exponentially with time in some region of the phase space.

Consider small region $\Omega$ of the phase space near the point $\left(\vec{q}_{0}, \vec{p}_{0}\right)$. Suppose that there are two classical trajectories $\left(\vec{q}^{(1)}(t), \vec{p}^{(1)}(t)\right)$ and $\left(\vec{q}^{(2)}(t), \vec{p}^{(2)}(t)\right)$ in $\Omega$. Then deviations of trajectories are

$$
\delta \vec{q}(t)=\vec{q}^{(1)}(t)-\vec{q}^{(2)}(t) \quad, \quad \delta \vec{p}(t)=\vec{p}^{(1)}(t)-\vec{p}^{(2)}(t)
$$

Evolution of these deviations in $\Omega$ is governed by the following linearized Hamilton equations

$$
\frac{d}{d t}\left(\begin{array}{c}
\delta \vec{q} \\
\delta \vec{p}
\end{array}\right)=G\left(\begin{array}{c}
\delta \vec{q} \\
\delta \vec{p}
\end{array}\right) \quad, \quad G \equiv\left(\begin{array}{cc}
0 & I \\
-\Sigma & 0
\end{array}\right)
$$

Here $I$ is the $N \times N$ identity matrix, $G$ is a stability matrix and matrix $\Sigma$ is

$$
\Sigma \equiv\left(\left.\frac{\partial^{2} V}{\partial q_{i} \partial q_{j}}\right|_{\vec{q}_{0}}\right)
$$

Matrix $\Sigma$ and stability matrix $G$ are functions of the point $\vec{q}_{0}$ of configuration space of the system. Solution of the equations (3) valid in $\Omega$ has the following form

$$
\left(\begin{array}{c}
\delta \vec{q}(t) \\
\delta \vec{p}(t)
\end{array}\right)=\sum_{i=1}^{2 N} C_{i} \exp \left\{\lambda_{i} t\right\}\left(\begin{array}{c}
\delta \vec{q}(0) \\
\delta \vec{p}(0)
\end{array}\right) .
$$

Here $\lambda_{i}=\lambda_{i}\left(\vec{q}_{0}\right)$ are eigenvalues of the stability matrix $G$. And $\left\{C_{i}\right\}$ is a full set of projectors. From (5) it is seen:

a) If there is $i$ such as $\operatorname{Re} \lambda_{i}>0$ then the distance between neighboring trajectories grows exponentially with time and motion is locally unstable. According Liouville's theorem stretching of phase space flow in one direction $\left(\operatorname{Re} \lambda_{i}>0\right)$ is accompanied by its compression in other direction (directions) in order to keep phase space volume constant. That means the existence of $\operatorname{Re} \lambda_{j}<0$. Thus for local instability of motion we can demand existence of $\operatorname{Re} \lambda_{k} \neq 0$. 
b) If for any $i=\overline{1,2 N} \quad \operatorname{Re} \lambda_{i}=0$ then there is no local instability and the motion is regular.

It is easy to see that $G^{2}=\operatorname{diag}(-\Sigma,-\Sigma)$. Therefore if $\left(-\xi_{i}\right), i=\overline{1, N}$ are eigenvalues of the matrix $(-\Sigma)$ then

$$
\left(-\xi_{i}\right)=\lambda_{i}^{2}, i=\overline{1, N} \quad, \quad \lambda_{i}^{2}=\lambda_{i+N}^{2}, i=\overline{1, N} .
$$

Thus without loss of generality we can imply that $\operatorname{Re} \lambda_{i} \geq 0$. Notice that

$$
\xi_{i}=-\lambda_{i}^{2}=\left(\operatorname{Im} \lambda_{i}\right)^{2}-\left(\operatorname{Re} \lambda_{i}\right)^{2}-2 i \operatorname{Im} \lambda_{i} \operatorname{Re} \lambda_{i}, i=\overline{1, N}
$$

Since matrix $\Sigma$ is real and symmetric its eigenvalues $\left\{\xi_{i}\right\}, \quad i=\overline{1, N}$ are real. Therefore (可) leads to the expression

$$
\operatorname{Im} \lambda_{i} \operatorname{Re} \lambda_{i}=0 \quad \forall i=\overline{1, N}
$$

Thus any eigenvalue of the stability matrix $G$ is real or pure imaginary or equals zero. Therefore the generalized Toda criterion for classical Hamiltonian systems with any finite number of freedoms can be formulated as follows:

a) If $\xi_{i} \geq 0, \forall i=\overline{1, N}$ then behavior of the system is regular near the point $\vec{q}_{0}$.

b) If $\exists i=\overline{1, N}: \xi_{i}<0$ then behavior of the system is locally unstable near the point $\vec{q}_{0}$.

If one of these conditions holds in some region of the configuration space then the motion is stable or chaotic respectively in this region. These results for systems with two degrees of freedom coincide with ones obtained in [15].

Now we give some qualitative arguments which bring us to formulation of chaos criterion in quantum mechanics and quantum field theory. From statistical mechanics and ergodic theory it is known that chaos in classical systems is a consequence of the property of mixing [4]-66]. Mixing means rapid (exponential) decrease of correlation function with time [6]. In other words, if correlation function exponentially decreases than the corresponding motion is chaotic, if it oscillates or is constant then the motion is regular [18]. We expand criterion of this type for quantum field systems. All stated bellow remains valid for quantum mechanics, since mathematical description via path integrals is the same.

For field systems the analogue of classical correlation function is two-point connected Green function

$$
G_{i k}(x, y)=-\left.\frac{\delta^{2} W[\vec{J}]}{\delta J_{i}(x) \delta J_{k}(y)}\right|_{\vec{J}=0}
$$

Here $W[\vec{J}]$ is generating functional of connected Green functions, $\vec{J}$ are the sources of the fields, $x, y$ are 4 -vectors of space-time coordinates.

Thus we formulate chaos criterion for quantum field theory and quantum mechanics in the following form:

a) If two-point Green function (9) exponentially goes to zero when the distance between its arguments goes to infinity then system is chaotic.

b) If it oscillates or remains constant in this limit then we have regular behavior of quantum system. 
To check the agreement between generalized Toda criterion and formulated quantum chaos criterion in semi-classical limit we shall calculate two-point Green function in semiclassical approximation of quantum mechanics. Generating functional is

$$
Z[\vec{J}]=\int D \vec{q} \exp \left\{i \int_{-\infty}^{+\infty} d t\left[\frac{1}{2} \dot{\vec{q}}^{2}-V(\vec{q})+\vec{J}^{T} \vec{q}\right]\right\}
$$

Here index $T$ denotes transposition. Consider certain solution of classical equations of motion $\vec{q}_{0}(t)$. Introduce new variable describing deviations from the classical trajectory $\delta \vec{q}(t)=$ $\vec{q}-\vec{q}_{0}(t)$, then under semi-classical approximation

$$
Z[\vec{J}]=\exp \left\{i S_{0}[\vec{J}]\right\} \int D \delta \vec{q} \exp \left\{i \int_{-\infty}^{+\infty} d t\left[\frac{1}{2} \delta \dot{\vec{q}}^{2}-\frac{1}{2} \delta \vec{q}^{T} \Sigma \delta \vec{q}+\vec{J}^{T} \delta \vec{q}\right]\right\} .
$$

Here classical action

$$
S_{0}[\vec{J}]=\int_{-\infty}^{+\infty} d t\left[\frac{1}{2} \dot{\vec{q}}_{0}^{2}-V\left(\vec{q}_{0}(t)\right)+\vec{J}^{T} \vec{q}_{0}\right]
$$

and matrix $\Sigma$ defined in (4) is real and symmetric. Therefore there exists orthogonal transformation $O$ reducing $\Sigma$ to diagonal form. For simplicity we suppose that $\xi_{i}=\xi_{i}\left(\vec{q}_{0}(t)\right), \quad i=$ $\overline{1, N}$ do not depend on time and remain constant on the classical trajectory. After orthogonal transformation given by $\delta \vec{q}=O \vec{x}, \quad \vec{J}^{T}=\vec{\eta}^{T} O^{T}, \quad O^{T} \Sigma O=\operatorname{diag}\left(\xi_{1}, \ldots, \xi_{N}\right) ; \quad O^{T} O=$ $O O^{T}=I$ we obtain

$$
\begin{gathered}
Z[\vec{\eta}]=L \exp \left\{i S_{0}[\vec{\eta}]\right\} \times \\
\int D \vec{x} \exp \left\{i \int_{-\infty}^{+\infty} d t\left(\frac{1}{2} \dot{\vec{x}}^{2}-\frac{1}{2} \vec{x}^{T} \operatorname{diag}\left(\xi_{1}, \ldots, \xi_{\mathrm{N}}\right) \overrightarrow{\mathrm{x}}+\vec{\eta}^{\mathrm{T}} \overrightarrow{\mathrm{x}}\right)\right\}
\end{gathered}
$$

here $L$ denotes Jacobian of the orthogonal transformation. After analytical extension of generating functional (13) into Euclidian space and path integration we get

$$
\begin{gathered}
Z_{E}[\vec{\eta}]=N \exp \left(-S_{0}^{E}[\vec{\eta}]\right) \times \\
\times \prod_{i=1}^{N} \exp \frac{1}{2} \int_{-\infty}^{+\infty} d \tau_{1} d \tau_{2} \eta_{i}\left(\tau_{1}\right)\left[\delta\left(\tau_{1}-\tau_{2}\right)\left(-\frac{d^{2}}{d \tau_{2}^{2}}+\xi_{i}\right)\right]^{-1} \eta_{i}\left(\tau_{2}\right) .
\end{gathered}
$$

Here and further there is no sum by $i, \mathrm{~N}$ is a normalization factor. Classical Euclidian action $S_{0}^{E}[\vec{\eta}]$ is a linear functional of the sources $\vec{\eta}$. Inverse operator

$$
\Delta_{i}\left(\tau_{1}, \tau_{2}\right)=\left[\delta\left(\tau_{1}-\tau_{2}\right)\left(-\frac{d^{2}}{d \tau_{2}^{2}}+\xi_{i}\right)\right]^{-1}
$$

has to satisfy the following equation

$$
\left(-\frac{d^{2}}{d \tau_{1}^{2}}+\xi_{i}\right) \Delta\left(\tau_{1}, \tau_{2}\right)=\delta\left(\tau_{1}-\tau_{2}\right)
$$


The solution is

$$
\Delta_{i}\left(\tau_{1}, \tau_{2}\right)=\frac{1}{2 \pi} \int d \omega \frac{e^{i \omega\left(\tau_{1}-\tau_{2}\right)}}{\omega^{2}+\xi_{i}} .
$$

Euclidian connected two-point Green function equals $\Delta_{i}\left(\tau_{1}, \tau_{2}\right)$. Its analytical extension to real (physical) time is

$$
G_{i}\left(t_{1}, t_{2}\right)=\frac{i}{2 \pi} \int d \varpi \frac{e^{i \varpi\left(t_{1}-t_{2}\right)}}{\varpi^{2}+\lambda_{i}^{2}},
$$

here $\tau \rightarrow i t, \omega \rightarrow-i \varpi$. Green function is defined up to any solution of corresponding homogeneous equation. We use this freedom to make Green function finite in the limit $\left(t_{1}-t_{2}\right) \rightarrow+\infty$ for real $\lambda_{i}$ and to obtain single formula for any $\lambda_{i}$ (both real and imaginary). Thus two-point connected Green function (18) can be represented in the form

$$
G_{i}\left(t_{1}, t_{2}\right)=\frac{i}{2} \operatorname{Re}\left(\frac{e^{-\lambda_{i}\left(t_{1}-t_{2}\right)}}{\lambda_{i}}\right), \quad t_{1}>t_{2} .
$$

From the expression (19) it is seen

a) If classical motion is locally unstable (chaotic) then according Toda criterion there is real eigenvalue $\lambda_{i}$. Therefore Green function (19) exponentially goes to zero for some $i$ when $\left(t_{1}-t_{2}\right) \rightarrow+\infty$. Opposite is also true. If Green function (19) exponentially goes to zero under the condition $\left(t_{1}-t_{2}\right) \rightarrow+\infty$ for some $i$, then there exists real eigenvalue of the stability matrix and thus classical motion is locally unstable.

b) If all eigenvalues of the stability matrix $G$ are pure imaginary, that corresponds classically stable motion, then in the limit $\left(t_{1}-t_{2}\right) \rightarrow+\infty$ Green function (19) oscillates as a sine. Opposite is also true. If for any $i$ Green functions oscillate in the limit $\left(t_{1}-t_{2}\right) \rightarrow+\infty$ then $\left\{\lambda_{i}\right\}$ are pure imaginary for any $i$ and classical motion is stable and regular.

Thus we have demonstrated that proposed quantum chaos criterion coincides with Toda criterion in the semi-classical limit (corresponding principle).

One of possible applications of proposed chaos criterion in field theory is an investigation of the stability of classical solutions with respect to small perturbations of initial conditions. Of course, this does not directly imply chaos, but advances us to it. To study the stability of certain classical solution of field equations one has to calculate (for instance, using one loop approximation) two-point Green function in the vicinity of considered classical solution.

To demonstrate this, consider real scalar $\varphi^{4}$-field

$$
L=\frac{1}{2}\left(\partial_{\mu} \varphi\right)^{2}-\frac{1}{2} m^{2} \varphi^{2}-\frac{\lambda}{4 !} \varphi^{4} .
$$

Here $\lambda>0$ is a coupling constant, $m^{2}$ is some parameter which can be larger or less then zero. In both cases $\varphi=0$ is a solution of field equations. Asymptotic of two-point Green function calculated in the vicinity of the classical solution $\varphi=0$ in the zero order of perturbation theory is

$$
G(x, y)_{\rho \rightarrow \infty}^{\sim} \rho^{-\frac{1}{2}} e^{i m \sqrt{\rho}} .
$$

Here $\rho=(x-y)^{2}$ and we accept that 4 -vector $x-y$ is inside the light cone $\left(x^{0}-y^{0}\right)>0$, in other words $\rho>0$. We can study the stability of considered solution with respect to small perturbations. Expression (21) shows that we have two different cases 
a) Green function oscillates and slowly (non-exponentially) goes to zero when $\rho \rightarrow \infty$. According proposed chaos criterion considered solution is stable. Indeed, from (21) it follows that parameter $m$ is real in this case. Therefore $\varphi=0$ is a stable vacuum state.

b) Green function exponentially goes to zero in the limit $\rho \rightarrow \infty$. From proposed chaos criterion it follows that $\varphi=0$ is an unstable solution. That is true since from (21) one can see that parameter $m$ has to be pure imaginary. It is known that in this case state $\varphi=0$ becomes unstable, two new stable vacuums are appeared and we obtain spontaneous symmetry breakdown 19 .

Thus in this paper we have generalized Toda criterion for the Hamiltonian systems with any finite number of degrees of freedom. Basing on the formal analogy between statistical mechanics and quantum field theory we proposed chaos criterion for quantum mechanical and quantum field systems. We have demonstrated that proposed chaos criterion corresponds to generalized Toda criterion in semi-classical limit of quantum mechanics in the case when Lyapunov exponents do not depend on time. For real scalar $\varphi^{4}$-field we analysed the stability of vacuum state and showed that spontaneous symmetry breakdown and degeneration of vacuum state can be regarded as signatures of quantum chaos. 


\section{References}

[1] A.N. Kolmogorov, Reports AS USSR, 98 (1954), p.527 (in Russian).

[2] V.I. Arnold, Izvestiya Akad. Nauk SSSR 25 (1961) p.21 (in Russian).

[3] N.S. Krylov, Works on substantiation of statistical physics.- Izdatelstvo Akad. Nauk SSSR, Moskow-Leningrad, 1950 (in Russian).

[4] A.J. Lichtenberg, M.A. Lieberman, Regular and Stochastic Motion.- Springer-Verlag, New York, 1983.

[5] G.M. Zaslavskii, R.Z. Sagdeev, Introduction in nonlinear physics.-Moskow, Nauka, 1988 (in Russian).

[6] G.M. Zaslavskii, Stochasticity of dynamical systems.-Moskow, Nauka, 1984 (in Russian).

[7] T. Prosen, M. Robnik, J. Phys. A, 27 (1994), p.8059; B.V. Chirikov, proceed. of II Int. Workshop on Squeezed States and Uncertainty Relations, P.N. Lebedev Phys. Inst., Moskow, May 25-29 (1992), NASA Conf. Publ. 3219, p. 317.

[8] B. Li, M. Robnik, J. Phys. A, 28 (1995), p.4843.

[9] T. Kawabe, S. Ohta, Phys. Rev. 44D (1991), p.1274.

[10] T.S. Biro, B. Muller, S.G. Matinyan, hep-th/0010134, 2000.

[11] V.E. Bunakov, proceed. of the XXXII Winter School of PNPI, Feb. 22-28 (1998).

[12] G. Mandelbaum, proceed. of Int. Conf. "Chaos and Complexity".- Edition Frontieres (1995) p.193.

[13] E.A. De Wolf, I.M. Dremin, W. Kittel, Phys. Rep. 270 (1996), p.1.

[14] M. Toda, Phys. Lett. A48 (1974), p.335.

[15] L. Salasnich, proceed. of the VIII Int. Conf. on Symm. Meth. in Phys., Dubna (1997); nucl-th/9707035, 1997.

[16] O.G. Sitenko, V.K. Tartakovski, Theory of Nucleai.-Kiev, Libid, 2000 (in Ukrainian).

[17] V.I. Kuvshinov, A.V. Kuzmin, Nonl. Phenom. in Complex Syst., vol. 4, num.1 (2001) p.64.

[18] H.G. Schuster, Detrministic Chaos: An Introduction.-- Physik-Verlag Weinheim, 1984.

[19] P.W. Higgs, Phys. Rev. Lett. 12 (1964), p. 162. 